\title{
Penile Erysipelas: A Study of 14 Cases
}

\section{Fatma Frikha, MD, Emna Bahloul, MD, Abderrahmen Masmoudi, Ph.D, Madiha Mseddi, Ph.D Meriem Amouri, Ph.D, Hamida Turki, Ph.D}

Department of Dermatology, Hedi Chaker University Hospital, Sfax, Tunisia.

\section{frikhafat@gmail.com}

\begin{abstract}
Introduction: Penile erysipelas is rarely described and not well characterized. Therefore we aim to describe the clinical characteristics, and risk factors of this erysipelas type.

Methods: a retrospective study of all clinically diagnosed cases of penile erysipelas during 18 years. For each patient, epidemiologic, clinical and therapeutic profiles were reported.

Results: fourteen cases of penile erysipelas were diagnosed ( $0.67 \%$ of all erysipelas patients). Mean age was 49 years. All patients had erythema, edema and pain in the penile region. Scrotal involvement was seen in 3 cases. Only one patient had phlyctenular lesions. The inguinal lymph nodes were enlarged bilaterally and painful on palpation in 13 cases (93\%). This cellulitis was a consequence of balanitis due to incomplete circumcision ( 2 cases), to sexual trauma ( 2 cases), direct trauma ( 2 cases), folliculitis (1 case) and inguinal intertrigo (1 case). Antibiotics were sufficient to obtain recovery in 13 cases. One of the patients, developed gangrene and underwent surgical operation.
\end{abstract}

Conclusion: Penile erysipelas looks to be favored by poorly done circumcision and micro trauma of the penis. Appropriate antibiotics must be administrated as soon as possible to prevent evolution into gangrene.

Keywords: Erysipelas, Cellulitis, erysipelas penis, incomplete circumcision, antibiotics

\section{INTRODUCTION}

Erysipelas is a frequent, acute bacterial infection of the dermis and hypodermis. The most important local pathogenic factor is a disruption of the epidermal barrier that serves as portal of entry for bacteria, thus determining the localization of erysipelas. The most common localizations of the disease are the lower extremities, face and trunk. Erysipelas of the genital region occurs in $0.5-2 \%$ (1). Penile erysipelas is unusual and often unrecognized. Our objective is to elucidate the epidemiological and clinical features, management and evolutional aspects of penile erysipelas in our region.

\section{Patients And Methods}

We conducted a retrospective study in the department of Dermatology during 18years (between 2000 and 2017), including all cases of penile erysipelas. Patients described in our study were seen during the clinical routine, and erysipelas was diagnosed by a dermatologist based on clinical aspect. For each patient, epidemiologic, clinical and therapeutic profiles were reported.

\section{RESULTS}

Fourteen cases of penile erysipelas were included, which represent 0,67\% of all cases of erysipelas during the same period. Mean age of patients was 49 years (17-67 years). The average time between onset of symptoms and diagnosis was 2.2 days. No patient had predisposing systemic disease. Portal of entry for bacteria were 
found in 8 cases (57\%) (incomplete circumcision ( 2 cases), sexual trauma (2 cases), direct trauma (2 cases), folliculitis ( 1 case) and inguinal intertrigo (1 case)). Erythema, edema and pain in the penile region (100\%) associated with fever $(100 \%)$ and chills $(78,6 \%)$ were the main symptoms in all cases (table 1) (Figure 1). Scrotal involvement was seen in 3 cases and mons pubis involvement in 1 case (Figure 1 and 2). Only one patient had bullous lesions. No patient described urinary symptoms. The inguinal lymph nodes were enlarged and painful on palpation in 13 cases (93\%). Laboratory tests, made for hospitalized patients (6 cases: 43\%), showed elevated serum levels of C-reactive protein and elevated leukocyte counts in all cases. Microbiological test, performed in 6 cases, was positive for Streptococcus pyogenes in only one case.

Initial antibiotic therapy was intravenously applied in an inpatient setting in standard dosages in only 6 patients. In 8 cases, antibiotic therapy was administrated in an outpatient setting. In most patients (12 cases: 85,7\%) a monotherapy was sufficient. Two patients received a combination therapy of Amoxicillin plus clavulanic acid and ciprofloxacin (table 1). Mean duration of treatment was 12,8 days (10 days-1 month). The skin of 13 patients was completely or almost completely cleared by the end of the antibiotic therapy. One patient, who had eythemobullous erysipelas developed gangrene (Figure 3) and underwent surgical treatment.

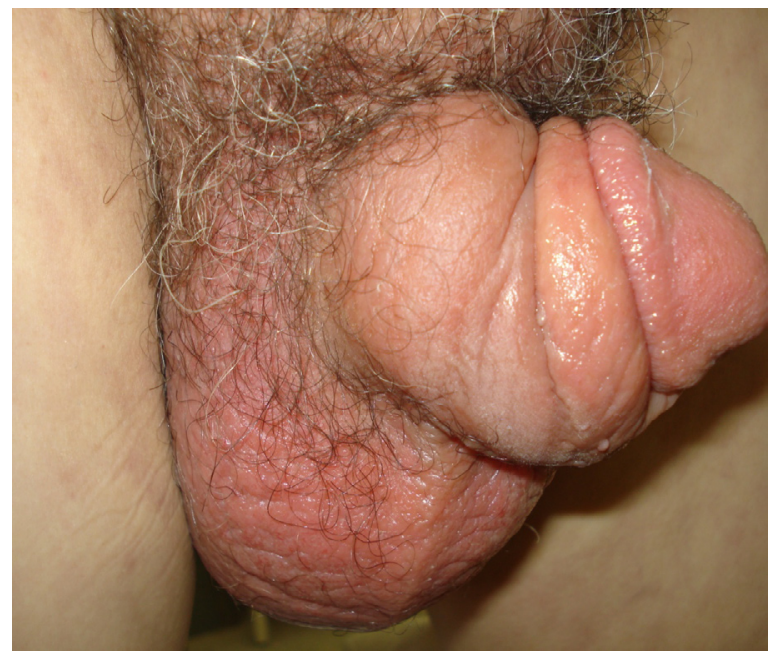

Fig1. Erythema and edema of the penis and scrotums

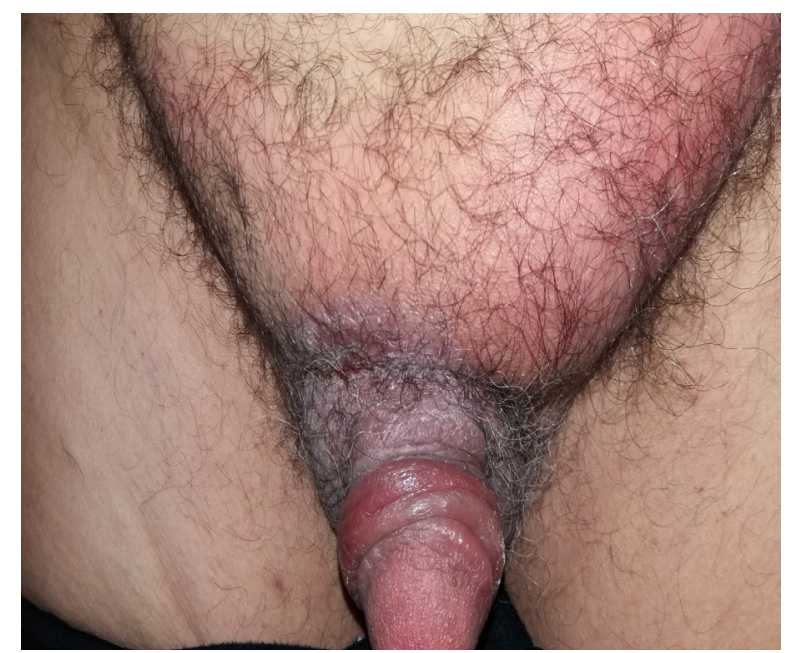

Fig2. Erythema and edema of the penis, scrotums and the mons pubis

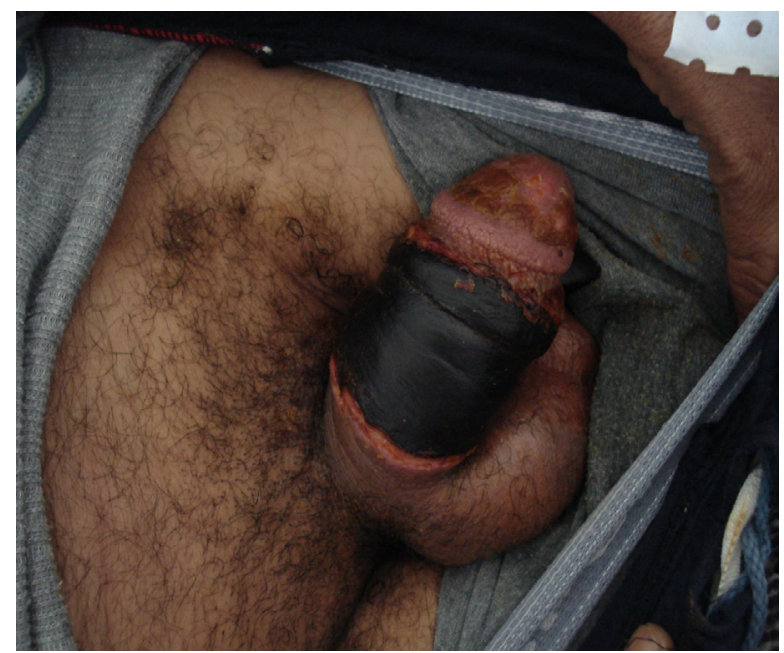

Fig3. Gangrene of the penis succeeding erythemobullous erysipelas 
Penile Erysipelas: A Study of 14 Cases

Table1. Main clinical characteristics, laboratory tests and therapy of patients with penile erysipelas

\begin{tabular}{|c|c|}
\hline Parameter & Number of patients (\%) \\
\hline Portal of entry & $8(57,1)$ \\
\hline *incomplete circumcision & $2(14,3)$ \\
\hline *sexual trauma & $2(14,3)$ \\
\hline *direct trauma of the penis & $2(14,3)$ \\
\hline$*_{\text {folliculitis }}$ & $1(7,1)$ \\
\hline *inguinal intertrigo & $1(7,1)$ \\
\hline \multicolumn{2}{|l|}{ Extra cutaneous signs } \\
\hline Fever & $14(100)$ \\
\hline Chills & $11(78,6)$ \\
\hline Pain & $14(100)$ \\
\hline \multicolumn{2}{|l|}{ Cutaneous examination } \\
\hline Erythema and edema of the penis & $14(100)$ \\
\hline Scrotal involvement & $3(21,4)$ \\
\hline Mons pubis involvement & $1(7,1)$ \\
\hline Bullous lesions & $1(7,1)$ \\
\hline Local complication (gangrene) & $1(7,1)$ \\
\hline Inguinal lymphadenopathy & $13(93)$ \\
\hline Laboratory tests & $6(42,8)$ \\
\hline C-reactive protein elevation & $6(100)$ \\
\hline Leukocytosis & $6(100)$ \\
\hline Positive microbiolgical culture & $1(16,7)$ \\
\hline \multicolumn{2}{|l|}{ Antibiotic therapy } \\
\hline Monotherapy & $12(85,7)$ \\
\hline *Amoxicillin plus clavulanic acid & $7(50)$ \\
\hline * Pristinamycin & $4(28,5)$ \\
\hline * Amoxicillin & $1(7,1)$ \\
\hline Combination of antibiotics & $2(14,3)$ \\
\hline *Amoxicillin plus clavulanic acid + ciprofloxacin & $2(14,3)$ \\
\hline
\end{tabular}

American Research Journal of Dermatology 


\section{DISCUSSION}

The predilection site of erysipelas in $80-90 \%$ of all cases is the lower leg (2) (3). Erysipelas occurs on the face in $2.5-19 \%$, the arm in 2-9\%, or the genital region in $0.5-2 \%$ (1) (2)Penile erysipelas is a rare entity but its real frequency seems to be underestimated. It is a clinical form of acute cellulitis (4). Penile erysipelas is a clinical condition that affects mainly adults, but can affect all ages (5) (6). Predisposing factors are mainly genital elephantiasis and systemic risk factors (liver and kidney disease, heart failure, neoplasms, immunosuppression, diabetes, hyperuricemia, hyperlipidemia, hypertension, obesity, seated position, use of nonsteroidal antiinflammatory drugs, smoking, and alcohol abuse (7) (8).

Different traumas and wounds, as well as existing dermatoses in the genital area (mycoses, balanoposthitis, and genital herpes) are necessary for the development of infection (9). Erysipelas of the penis usually presents with penile swelling and pain, and may be associated with discharge, fever and inguinal lymphadenopathy, like in our cases (10) (4). The diagnosis is based on the history, clinical findings, laboratory values and rarely microbiological culture results (11). The risk factors predisposing patients for local complications are not fully known (4). The necrotizing form of erysipelas has hemorrhagic and serous exudates in the buli with later skin necroses (11). Then, erythemobullous form of erysipelas represent a factor of poor prognosis as in our case (4). Appropriate antibiotics must be administrated as soon as possible to prevent evolution into gangrene. Naturally, Penicillin is the drug of first choice. Penicillin administered orally or intramuscularly is sufficient for most cases of classic erysipelas and should be given for duration of 10-20 days (11). If gangrene does develop, radical debridement of the necrotic tissue as well as a wide margin of adjacent inflamed skin must be undertaken (12).

\section{REFERENCES}

1. Glatz M, Degen D, French LE, Aberer W, Müllegger RR. Erysipelas of the thigh and the gluteal region: Retrospective multicenter analysis of a very rare entity in 39 patients. Dermatology. 2013;225(3):277-83.

2. Bonnetblanc J-M, B??dane C. Erysipelas. Am J Clin Dermatol. 2003;4(3):157-63.

3. Bernard P. Management of common bacterial infections of the skin. Curr Opin Infect Dis. 2008 Apr;21(2):122-8.

4. Titou H, Ebongo C, Bouati E, Boui M. Risk factors associated with local complications of erysipelas: a retrospective study of 152 cases. Pan Afr Med J. 2017;26:66.

5. Bliss DP, Healey PJ, Waldhausen JH. Necrotizing fasciitis after Plastibell circumcision.J Pediatr [Internet]. 1997 Sep [cited 2019 Jan 16];131(3):459-62. Available from: http://www.ncbi.nlm.nih.gov/pubmed/9329429

6. Brady MT. Cellulitis of the penis and scrotum due to group B streptococcus. J Urol. 1987 Apr;137(4):736-7.

7. Bartholomeeusen S, Vandenbroucke J, Truyers C, Buntinx F. Epidemiology and comorbidity of erysipelas in primary care. Dermatology. 2007;215(2):118-22.

8. Pereira de Godoy JM, Galacini Massari P, Yoshino Rosinha M, Marinelli Brandão R, Foroni Casas AL. Epidemiological data and comorbidities of 428 patients hospitalized with erysipelas. Angiology. $2010 \mathrm{Jul}$ 10;61(5):492-4.

9. Krasagakis K, Valachis A, Maniatakis P, Krüger-Krasagakis S, Samonis G, Tosca AD. Analysis of epidemiology, clinical features and management of erysipelas. Int J Dermatol [Internet]. 2010 Sep [cited 2016 Mar 20];49(9):1012-7. Available from: http://www.ncbi.nlm.nih.gov/pubmed/20931671 
Penile Erysipelas: A Study of 14 Cases

10. Jędrowiak A, Oszukowska M, Żuchowska A, Tabara K, Szewczyk A, Kuchciak-Brancewicz M, et al. Penoscrotal elephantiasis: Case report. Our Dermatology Online. 2017;8(2):204-6.

11. Bakardzhiev I. Erysipelas Penis. J IMAB - Annu Proceeding (Scientific Pap. 2011;17, 1(2011):146-8.

12. Haury B, Rodeheaver G, Stevenson T, Bacchetta C, Edgerton MT, Edlich RF. Streptococcal cellulitis of the scrotum and penis with secondary skin gangrene. Surg Gynecol Obstet. 1975 Jul;141(1):35-9.

Citation: Fatma Frikha, Emna Bahloul, Abderrahmen Masmoudi, et al. "Penile Erysipelas: A Study of 14 Cases". American Research Journal of Dermatology; 1(1): 33-37.

Copyright (c) Fatma Frikha, Emna Bahloul, Abderrahmen Masmoudi, et al. This is an open access article distributed under the Creative Commons Attribution License, which permits unrestricted use, distribution, and reproduction in any medium, provided the original work is properly cited. 